\title{
Permeability Calculation of Sand Conglomerate Reservoirs Based on Nuclear Magnetic Resonance (NMR)
}

\author{
You Zhou, ${ }^{1,2}$ Songtao Wu ${ }^{1},{ }^{1}$ Zhiping Li, ${ }^{3,4}$ Rukai Zhu, ${ }^{1}$ Shuyun Xie, ${ }^{5}$ Xiufen Zhai, \\ and Lei Lei $^{5}$ \\ ${ }^{1}$ PetroChina Research Institute of Petroleum Exploration and Development, Beijing 100083, China \\ ${ }^{2}$ School of Civil Engineering, Hebei University of Engineering, Handan 056000, China \\ ${ }^{3}$ School of Energy Resource, China University of Geosciences (Beijing), Beijing 100083, China \\ ${ }^{4}$ Beijing Key Laboratory of Unconventional Natural Gas Geological Evaluation and Development Engineering, Beijing 100083, China \\ ${ }^{5}$ Earth Science Faculty, China University of Geosciences (Wuhan), Wuhan 430000, China
}

Correspondence should be addressed to Songtao Wu; wust@petrochina.com.cn

Received 11 November 2019; Revised 22 July 2020; Accepted 4 August 2020; Published 28 September 2020

Academic Editor: Fabien Magri

Copyright (c) 2020 You Zhou et al. This is an open access article distributed under the Creative Commons Attribution License, which permits unrestricted use, distribution, and reproduction in any medium, provided the original work is properly cited.

\begin{abstract}
The concept of an intermingled fractal unit (IFU) model was first proposed by Atzeni and Pia in 2008, and their model has since been successfully applied to predict thermal conductivity, electrical conductivity, and the mechanical properties of porous media materials. This paper, based on the Pia IFU model, fits the pore size distribution spectrum to quantitatively characterize the Triassic Karamay Formation conglomerate reservoirs in the Mahu region, in the Junggar Basin of Northwest China, and makes permeability predictions using the free fluid $T_{2}$ spectrum according to the nuclear magnetic resonance (NMR) experimental data. The results show that the accuracy of the IFU model is significantly higher than that of the classic Coates and SDR models for conglomerate reservoirs with complex pore structures, indicating that this is an effective method to calculate permeability based on NMR. In addition, preliminary discussions are entered into regarding the intermingled fractal expression of the Kozeny-Carman equation and the relative permeability, in order to widen the application of the IFU model in reservoir physics. The derived expressions appear complicated in form but are straightforward to calculate and apply using computer programming since their iteration parameters are definite. The findings set out in this paper provide a valuable reference for further research of the IFU model in reservoir physics.
\end{abstract}

\section{Introduction}

The pore structure of reservoir rocks has fractal characteristics [1]. Because of this, a number of scholars have recently adopted fractal theory to establish the porosity or the permeability model of reservoir rocks and have achieved some productive results. For instance, Li et al. [2], Zheng et al. [3], and Bai et al. [4] have successfully applied fractal theory to build models for forecasting the permeability of tight sandstone reservoirs. With the development of fractal theory, however, it has become apparent that the theory has limitations in characterizing a real reservoir space. When computing fractal dimensions using capillary data, for example, it has been noted that in a $\log -\log$ coordinate system, the $\lg S_{\mathrm{Hg}}$ to $\lg P_{\mathrm{c}}$ plot sometimes exhibits multifractal characteristics for some kinds of rocks, instead of being linearly fitted, which significantly influences the accuracy of computation [5-9]. Meanwhile, Sima et al. [10] have pointed out that sand conglomerate rocks with high heterogeneity tend to exhibit more evident multifractal characteristics or even nonfractal characteristics, on log-log coordinates, illustrating that the probability of multifractal characteristics is greater in the conglomerate samples. This section analyzes the fractal feature gained from the capillary buddle subsection model and draws similar conclusions for sand conglomerate rocks (Figure 1). Figures 1(a)-1(c) show that the fractal characteristics of the capillary pressure curve of the sand conglomerate reservoir generally need to be fitted with two or more straight lines, which indicates that different intervals of pore size have different fractal dimensions. Figure 1(d) shows that the 


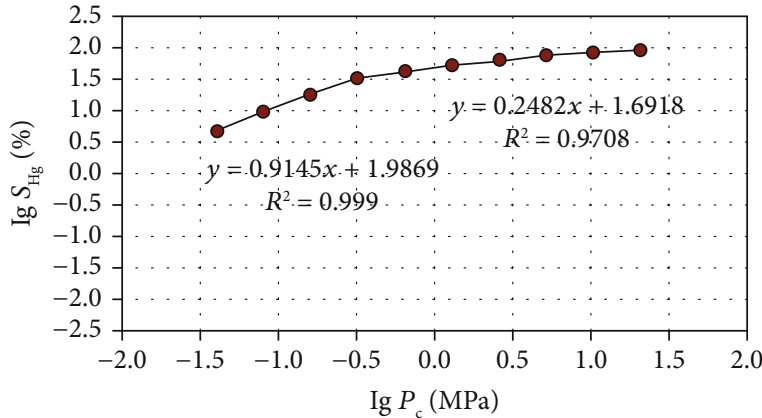

(a) Well X003

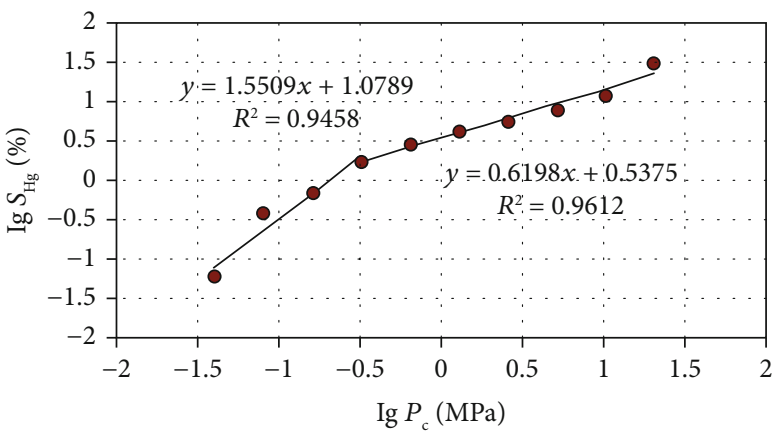

(c) Well H3

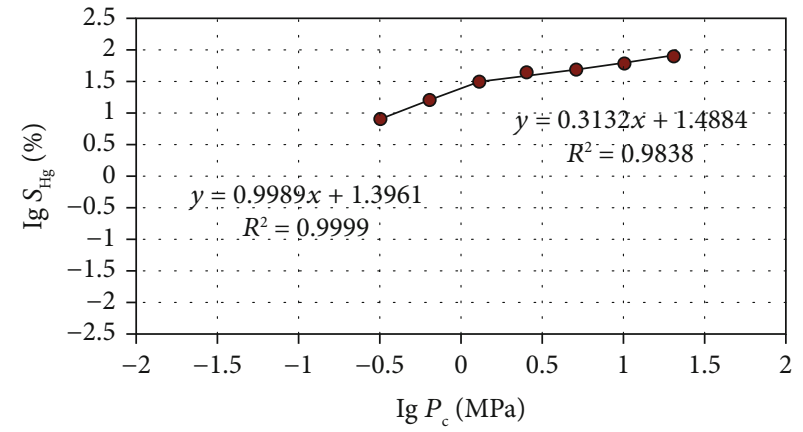

(b) Well M152

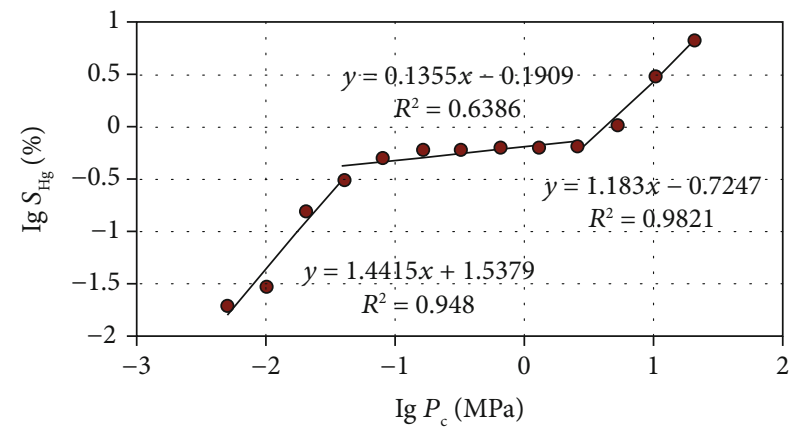

(d) Well H3

FIGURE 1: Multifractal and nonfractal characteristics of the capillary pressure curve of sand conglomerate reservoir.

fractal dimensions of some large intervals of pore size, calculated by the graphical method, are greater than 3 , and this phenomenon is interpreted as being attributable to nonfractal characteristics.

To address this problem, Li et al. [11] and Lai et al. [12] proposed to run a weighted average of the mercury saturation of each fractal segment to obtain a weighted fractal dimension. However, this method neglects the detailed features of the pore space and hence only reflects the overall fractal characteristics of rock pores. Moreover, although the fractal dimension is a highly significant parameter in fractal theory, it has limitations in characterizing a real rock pore space. This is because porous media with different pore structures may have the same or similar fractal dimension [13]. Figure 2 shows that although the pore structures of the four samples are different, their fractal dimensions tend to be similar. The specific calculation process for threedimensional fractal dimensions is set out by Zhang et al. [15]. Therefore, utilizing a single fractal dimension to establish a porosity or permeability model might inevitably constrain subsequent research on permeation performance and reservoir engineering.

Given the limitations of the single fractal dimension, Italian scholar Pia et al. [16-23] introduced an intermingled fractal unit (IFU) model, which has proven to be useful in simulating porous media. Pia and his research team applied the model to predict the thermal conductivity, electrical conductivity, mechanical properties, and permeability of porous media materials. Intuitively, the IFU model can be considered to be several superimposed fractal units. Each of the small fractal units in the IFU model is a modified Sierpiński carpet, with each fractal unit having different iteration parameters. Thus, the IFU model can be regarded as the Sierpiński Carpet modified by altering its iteration rule. The advantage of this model is that it can present detailed characterizations of internal spaces of complicated objects.

At present, applications of the IFU model have relatively little engagement in the field of petroleum engineering. Since Pia first used the IFU model to predict permeability based on mercury intrusion capillary pressure (MICP) data [20], some scholars have also done a number of works to widen the application of the model in recent years. Tan et al. [24] and Chen et al. [25] applied the IFU model to forecast the permeability with consideration of tortuosity according to the MICP data. Tan et al. [26] used X-ray computed tomography (CT) and scanning electron microscopy (SEM) data to make permeability prediction based on the model. Li et al. [27, 28] applied a 2D and 3D IFU model to predict permeability using SEM images for shales. Objectively, at present, the applications of the IFU model in reservoir physics basically focus on the prediction of permeability, and the IFU model based on NMR data has not been reported.

This paper, based on the Pia IFU model, constructs a model to fit the pore size distribution spectrum according to NMR measurements and makes permeability predictions using the free fluid $T_{2}$ spectrum. Meanwhile, some key issues in the process of modeling using NMR data are also pointed out. Also, new intermingled fractal expressions of the Kozeny-Carman equation and relative permeability are derived to broaden the application of the IFU model in reservoir physics. 


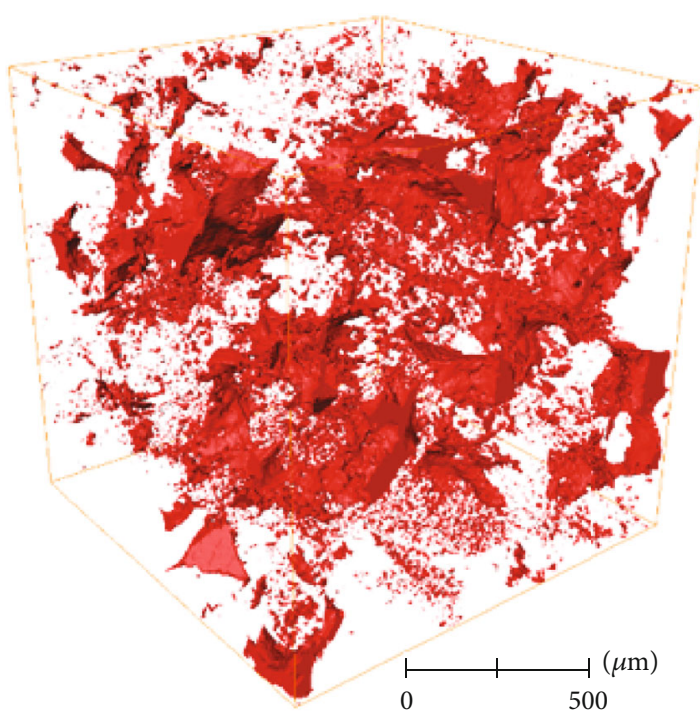

(a)

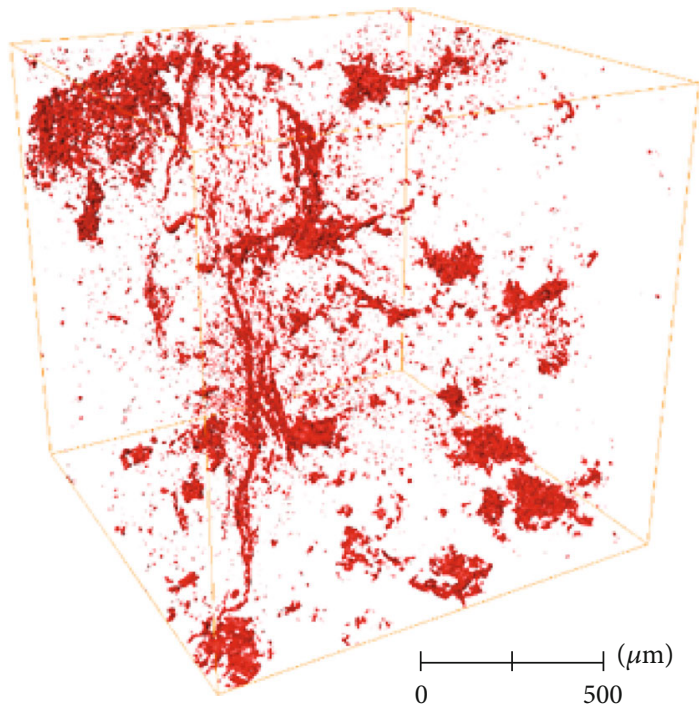

(c)

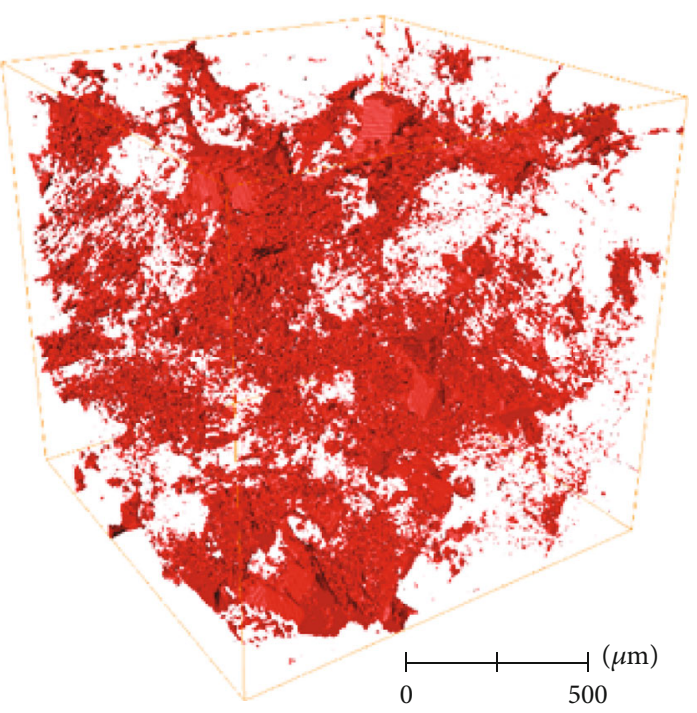

(b)

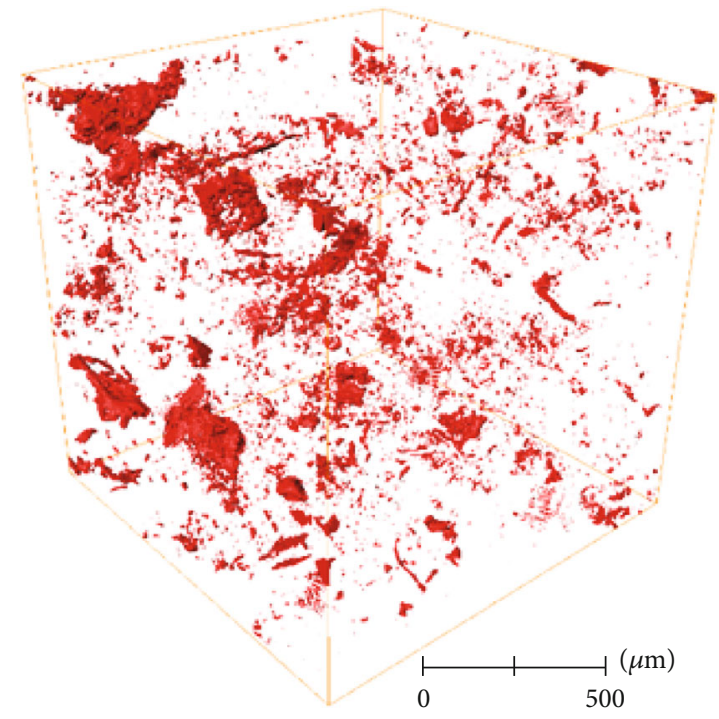

(d)

Figure 2: 3D pore model of micro-CT scanning and fractal dimensions for four typical sand conglomerate rock samples: (a) M5-4, fractal dimension = 2.66; (b) B64-33, fractal dimension = 2.64; (c) B64-49, fractal dimension = 2.21; (d) M101-1, fractal dimension = 2.20. Panel (b) was presented in our previous paper [14].

\section{Methodology}

2.1. Introduction to the Pia IFU Model. This section sets out some core steps for the derivation process of iterative formulas for the IFU model. For a detailed explanation of the entire process, see Pia et al. [20].

Without derogation from general principles, a specific IFU is used as an example to examine the relationships between its iteration parameters (Figure 3).

Let $d_{\max }$ be the maximum pore side length of this fractal unit. The side length of the fractal unit will then be $3 d_{\max } \cdot N_{\mathrm{p}}$ is the number of the child squares removed from each parent square in every iteration, and $b$ is the ratio factor (a multiple of equal divisions of the side length in each iteration). Thus, $N_{p}=1$ and $b=3 . N_{\text {soild }}$ is the number of the child squares that do not take part in the iteration. Thus, $N_{\text {soild }}=0$. The value $i$ is the iteration time.

According to fractal geometric theory [29], the fractal dimension $\left(D_{\mathrm{f}}\right)$ is the value of the number of squares that participate in the next iteration $\left(N_{\text {iteration }}\right)$ divided by the ratio factor $(b)$ :

$$
D_{\mathrm{f}}=\frac{\log \left(N_{\text {iteration }}\right)}{\log b} .
$$

For the Sierpiński carpet fractal model, the fractal dimension of the $3 \mathrm{D}$ spatial model is the value of the $2 \mathrm{D}$ plane model plus 1 . 


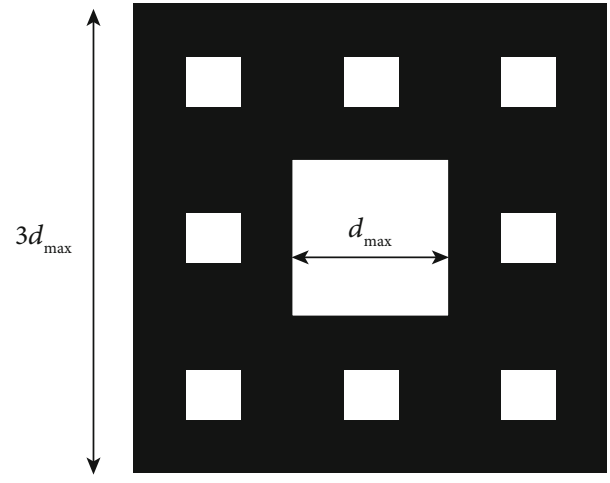

FIgURE 3: Schematic diagram of IFU.

To produce a Sierpiński carpet similar to the unit shown in Figure 3, the side length of the new pores formed in the $i$-th iteration is defined by Pia et al. [20] as follows:

$$
d_{i}=\frac{d_{\max }}{b^{i-1}} .
$$

The number of new pores formed in the $i$-th iteration $\left(N_{i}\right)$ is given by $\mathrm{Li}$ et al. [27]:

$$
N_{i}=\left(b^{2}-N_{\text {soild }}-N_{p}\right)^{i-1} \times N_{p} .
$$

For ease of theoretical calculation, it is assumed that the real percolation section of every square pore is its maximum incircle and that the diameter, position, and quantity of pores will be the same on any rock section when the $2 \mathrm{D}$ IFU model is extended to $3 \mathrm{D}$. If $L_{0}$ is the length of the model and the total iteration time for the fractal unit is $k$, then the generated total pore volume $V_{\mathrm{p}}$ is given as:

$$
V_{\mathrm{p}}=\sum_{i=1}^{k}\left[\left(b^{2}-N_{\text {soild }}-N_{\mathrm{p}}\right)^{i-1} \times N_{\mathrm{p}} \times \frac{\pi}{4}\left(\frac{d_{\max }}{b^{i-1}}\right)^{2} \times L_{0}\right] .
$$

The IFU model can then be utilized to reproduce the pore size distribution spectrum and porosity. The procedure used to reproduce porous microstructures is shown in Figure 4. For a detailed explanation of the process, see Pia's series of papers.

2.2. Permeability Calculation Using Traditional Classic Models Based on NMR Experiments. NMR experiments can quantitatively characterize the pore structures of rocks and can be applied directly to calculate permeability. The current classic models for calculating permeability using NMR data are the Timur-Coates and SDR models.

The Timur-Coates model [30] is expressed as follows:

$$
K=n_{1} \phi^{n_{2}}\left(\frac{\mathrm{FFI}}{\mathrm{BVI}}\right)^{n_{3}} \text {. }
$$

The SDR model [31] can be expressed as follows:

$$
K=m_{1}\left(\frac{\phi}{100}\right)^{m_{2}} T_{2 g} m_{3},
$$

where $K$ is the permeability $\left(10^{-15} \mathrm{~m}^{2}\right), \phi$ is the effective porosity (\%), $T_{2 \mathrm{~g}}$ is the geometric mean of $T_{2}$ (ms), FFI is the saturation of movable fluid (\%), BVI is the saturation of immovable fluid (\%), and $m_{1}, m_{2}, m_{3}, n_{1}, n_{2}$, and $n_{3}$ are the corresponding parameters of each model, which are determined by the results of actual core data used to fit experimental permeability. In the Coates model, in order to facilitate the calculation, the values of $n_{2}$ and $n_{3}$ are sometimes directly regarded as 4 and 2, respectively, as well as the values of $m_{2}$ and $m_{3}$ in the SRD model $[32,33]$.

In recent years, some scholars have pointed out that the classic Timur-Coates and SDR models have certain limitations for calculating the permeability of complex reservoirs. For example, the classic model has good applicability for the calculation of permeability in medium and high permeability reservoirs. However, for reservoirs with low porosity and low permeability, it is difficult to correlate the calculation results with experimental data [34]. This paper applied the classical model to predict the permeability of sand conglomerate reservoirs in the Mahu rim region of the Junggar Basin, and the calculation results exhibit significant discrepancies from the experimental results (see Section 3.2). This is because the sand conglomerate rocks have extremely high heterogeneity due to their complex gravel-cement configuration. By using micro-CT scanning technology on some typical sand conglomerate rock samples, it is indicated that the sand conglomerate rocks in the research area have complex and diverse microscopic pore structures (Figure 5).

Meanwhile, the actual calculations in the classic models are complicated, resulting in large volumes of calculations for the process of converting the NMR $T_{2}$ spectrum into a pseudocapillary pressure curve using the nonlinear method. Moreover, it is found that the conversion coefficients and conversion indices calculated by the classic model vary greatly, even for rock samples in the same study area. The average value of the region does not satisfy the requirement for calculation accuracy, and the nonlinear fitting method does not explain the theoretical relationship between $T_{2}$ spectral relaxation time and real pore sizes. Because of this, this paper utilizes the "centrifugation $T_{2 c}$ method" [35] to convert the $T_{2}$ spectrum into a corresponding pore size distribution for subsequent permeability prediction based on the IFU model.

There is an issue worth pointing out here. Since the sand conglomerates are easy to split in the process of centrifugal experiment, the cores are wrapped with a special heatshrinkable film in the experiments. Meanwhile, in order to determine the centrifugal force suitable for the samples in the study area, five representative samples were selected to test six different centrifugal forces of $100 \mathrm{psi}, 150 \mathrm{psi}$, $200 \mathrm{psi}, 250 \mathrm{psi}, 300 \mathrm{psi}$, and $350 \mathrm{psi}$. By analyzing the descend range of irreducible water saturation before and after centrifugation, it was found that until the centrifugal force 


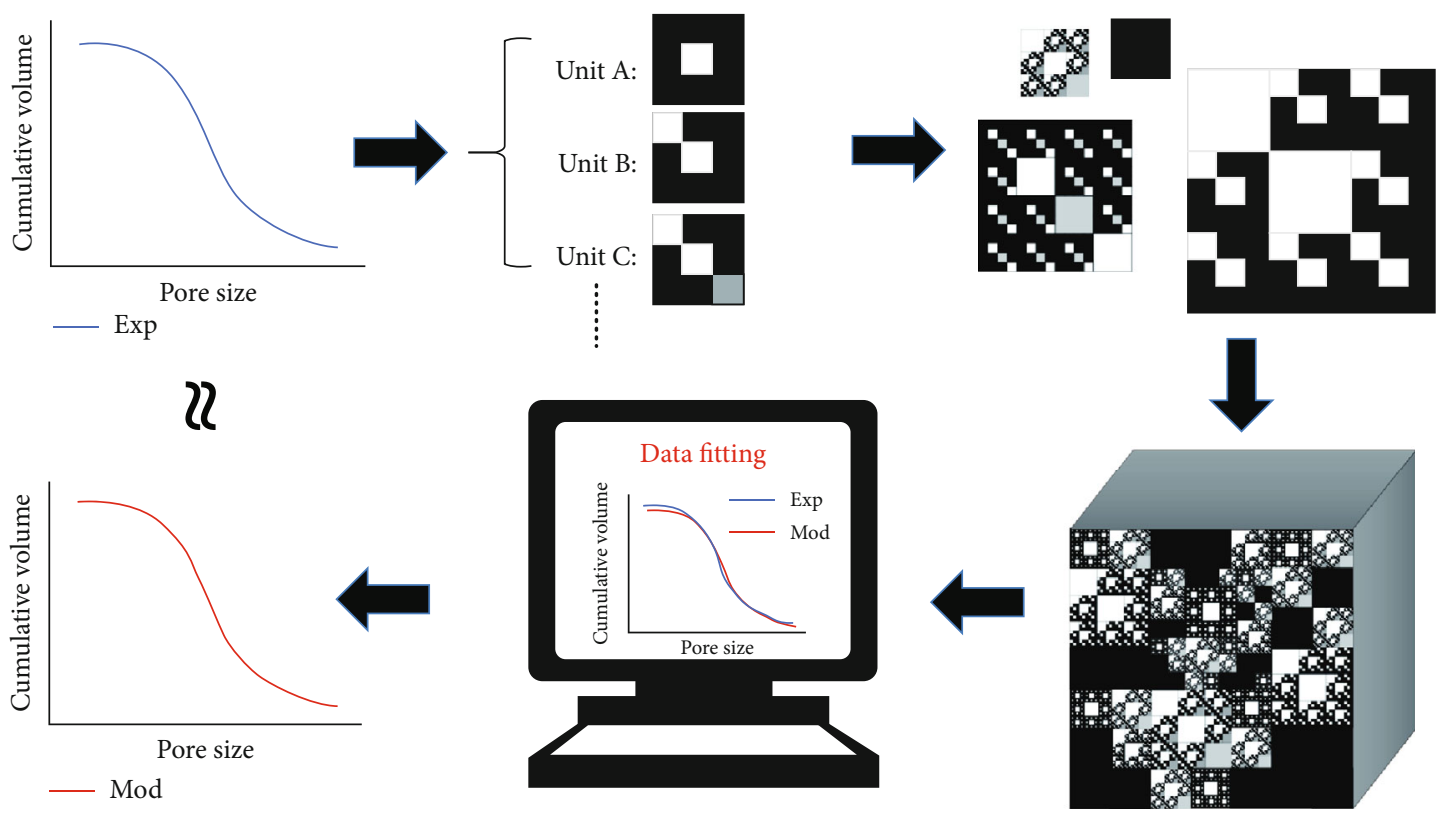

FIGURE 4: IFU procedure for the reproduction of porous microstructures (redrawn and modified based on an image from [20]).

increased from $300 \mathrm{psi}$ to $350 \mathrm{psi}$, the reduction of irreducible water saturation of five samples with different physical properties tended to be stable. Therefore, we select a centrifugal force of $300 \mathrm{psi}$ to calibrate a $T_{2}$ cutoff value, and then, the corresponding lower limit of the movable fluid throat radius is $0.0704 \mu \mathrm{m}$. The experiment adopts a GeoSpec2 NMR core analyzer produced by Oxford Instruments Magnetic Resonance in England. The working frequency is $2 \mathrm{MHz}$, the temperature of the instrument is $35^{\circ} \mathrm{C}$, the room temperature is $25^{\circ} \mathrm{C}$, and the humidity is $50 \%-60 \%$.

\subsection{Permeability Calculation Using the IFU Model Based on} NMR Experiments. The expression of permeability based on the IFU model is derived in this section.

The flow of a single pore formed after the $i$-th iteration is given by the Hagen-Poiseuille equation:

$$
q(d)=\frac{\pi d^{4} \Delta p}{128 \mu \tau L_{0}}
$$

where $q$ is the flow $\left(\mathrm{cm}^{3} / \mathrm{s}\right), \Delta p$ is the pressure difference between the ends of the capillary tube (atm), $\mu$ is the viscosity of fluid (mPa.s), $L_{0}$ is the characteristic length of the capillary tube $(\mathrm{cm})$, and $\tau$ is the tortuosity (dimensionless parameter). The ratio of the capillary tube's real length $\left(L_{t}\right)$ to the characteristic length $\left(L_{0}\right)$ is expressed:

$$
L_{\mathrm{t}}=\tau L_{0}
$$

Based on the average tortuosity-porosity analytical model for porous media consisting of square particles introduced by Yu et al. [36], Yun et al. [37] proposed an enhanced expres- sion of the average tortuosity of porous media consisting of circular particles, expressed as follows:

$$
\begin{aligned}
\tau= & 1-\frac{\phi}{2}+\frac{\sqrt{1-\phi}}{4} \\
& +\frac{(\phi+1+\sqrt{1-\phi}) \cdot \sqrt{9-5 \phi-8 \sqrt{1-\phi}}}{8 \phi},
\end{aligned}
$$

where $\phi$ is the porosity. Thus, the total flow $(Q)$ of the pores formed from the first to the $k$-th iteration is given as:

$Q=\sum_{i=1}^{k}\left(\left(\frac{\pi d_{\max }^{4} \Delta p}{128 b^{4(i-1)} \mu \tau L_{0}}\right) \cdot\left(\left(b^{2}-N_{\text {soild }}-N_{\mathrm{p}}\right)^{i-1} \times N_{\mathrm{p}}\right)\right)$.

According to the equivalent percolation principle,

$$
\begin{aligned}
& \sum_{i=1}^{k}\left(\left(\frac{\pi d_{\max }^{4} \Delta p}{128 b^{4(i-1)} \mu \tau L_{0}}\right) \cdot\left(\left(b^{2}-N_{\text {soild }}-N_{\mathrm{p}}\right)^{i-1} \times N_{\mathrm{p}}\right)\right) \\
& \quad=\frac{K A \Delta p}{\mu L_{0}} .
\end{aligned}
$$

Finally, the expression for computing permeability can be derived as follows:

$$
K=\frac{\pi d_{\max }^{4} \cdot N_{\mathrm{p}}}{128 \tau A} \sum_{i=1}^{k} \frac{\left(b^{2}-N_{\text {soild }}-N_{\mathrm{p}}\right)^{i-1}}{b^{4(i-1)}} .
$$

Thus, the permeability of a single fractal unit is given in formula (12), and the formula is similar in form to the 



FIGURE 5: Images showing the microscopic pore structure of some typical sand conglomerate reservoirs in the study area: (a-1-a-3) micro-CT $3 \mathrm{D}$ grayscale model, 3D pore model, and pore network model of reservoir M5-8, which is medium-coarse sandstone; (b-1-b-3) micro-CT 3D grayscale model, 3D pore model, and pore network model of reservoir M003-4, which is gravel-bearing coarse sandstone; (c-1-c-3) micro-CT $3 \mathrm{D}$ grayscale model, 3D pore model, and pore network model of reservoir M133-7, which is grain-supported conglomerate; (d-1-d-3) microCT 3D grayscale model, 3D pore model, and pore network model of reservoir B64-4, which is matrix-supported conglomerate.

expression in Pia et al. [20]. If the IFU model contains several fractal units, formulas (9) and (10) can be used to calculate its tortuosity and flow rates, respectively. The total permeability of the IFU model is easily obtained according to the equivalent seepage principle.

\section{Results and Discussion}

3.1. Conversion of the $T_{2}$ Spectrum to Pore Size Distribution. The basic principle of the "centrifugation $T_{2 c}$ method" is that the pore size $r_{0}$, corresponding to the cutoff value $T_{2 c}$ 


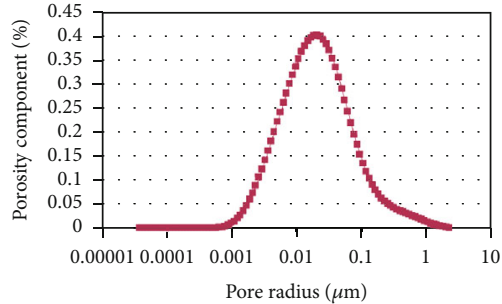

(a)

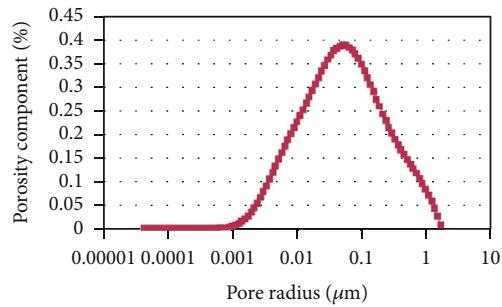

(d)

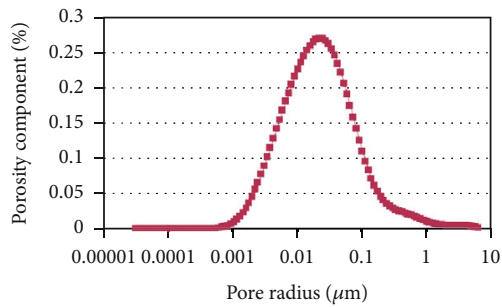

(g)

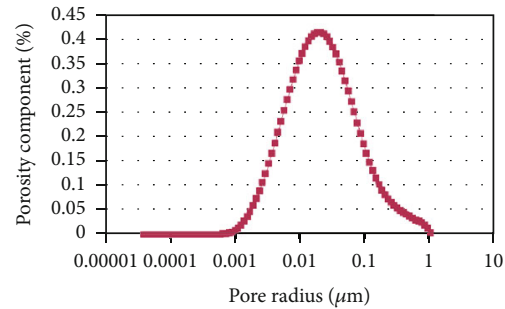

(b)

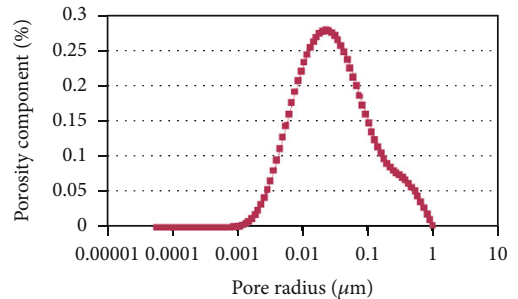

(e)

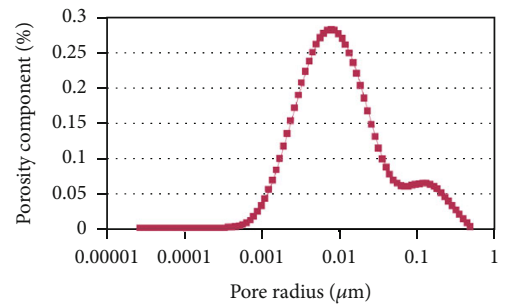

(h)

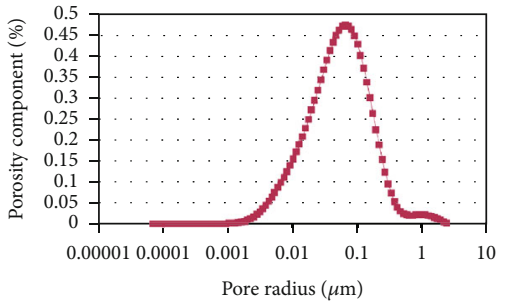

(c)

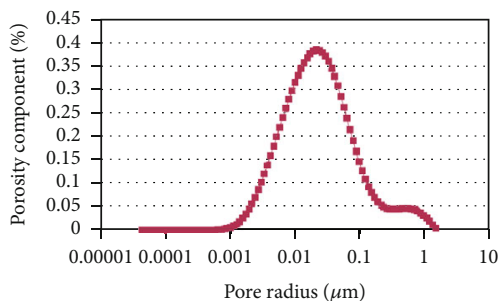

(f)

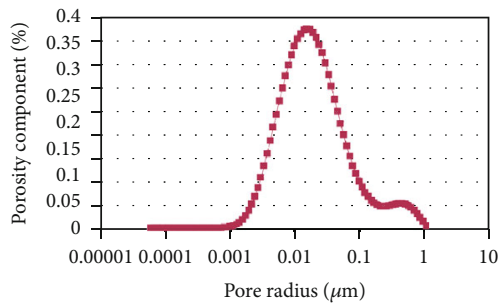

(i)

FIGURE 6: Conversion results of pore size distribution for some typical rock samples: (a) B64-3, (b) B64-29, (c) B64-33, (d) B64-38, (e) B64-42, (f) B65-8, (g) M5-1, (h) M5-6, and (i) M101-2-2.

determined by the centrifugal experiment, is a constant, and thence, the pore size $r_{i}$, corresponding to the $i$-th relaxation time $T_{2 i}$, is given as [38]

$$
r_{i}=\frac{r_{0} T_{2 i}}{T_{2 c}} .
$$

During the NMR experiment, the centrifugal pressure for testing is $300 \mathrm{psi}$, so the calculated pore size $r_{0}$ is $0.0704 \mu \mathrm{m}$, corresponding to $T_{2 c}$. By applying formula (13), the pore size corresponding to different relaxation times can be calculated, and the pore size distribution based on $T_{2}$ spectra can then be constructed as shown in Figure 6.

The $T_{2}$ spectrum of $100 \%$ saturated water is the reflection of all pore spaces in the rocks [39]; i.e., it contains connected pores, dead pores, and micropores storing bound water. Therefore, for permeability calculations based on NMR data, the free fluid $T_{2}$ spectrum should be used rather than the $100 \%$ saturated water $T_{2}$ spectrum. This is because only connected pores with percolation ability contribute to permeability in the pore space, and thus, only the free fluid $T_{2}$ spectrum has good correspondence with the real seepage space. The acquisition method for this value is to subtract the centrifugal $T_{2}$ spectrum signal from the saturated water $T_{2}$ spectrum sig- nal, and the remainder is the free fluid $T_{2}$ spectrum (Figure 7).

Also, an abnormality persists for some samples, in that the centrifugal $T_{2}$ spectrum in some samples is higher than the saturation $T_{2}$ spectrum (Figures 8(a) and 8(b)), resulting in the appearance of a "negative accumulation area" when the free fluid accumulation curve is obtained (Figures 8(c) and $8(d))$. These anomalies in the micropore intervals are difficult to avoid, because they are caused by system errors in the instrumentation. Therefore, when using the IFU model to fit the pore size distribution spectrum of the free fluid, it is suggested that the model is fitted to the highest point of the cumulative curve to circumvent system errors.

To summarize, the procedures of conversion of the $T_{2}$ spectrum to pore size distribution are as follows:

(1) Subtract the centrifugal $T_{2}$ spectrum from the $100 \%$ saturated water $T_{2}$ spectrum to obtain the free fluid $T_{2}$ spectrum

(2) Eliminate the "negative accumulation area" to obtain an effective free fluid accumulation curve

(3) Use formula (13) to convert the effective free fluid $T_{2}$ spectrum to the pore diameter cumulative distribution curve 


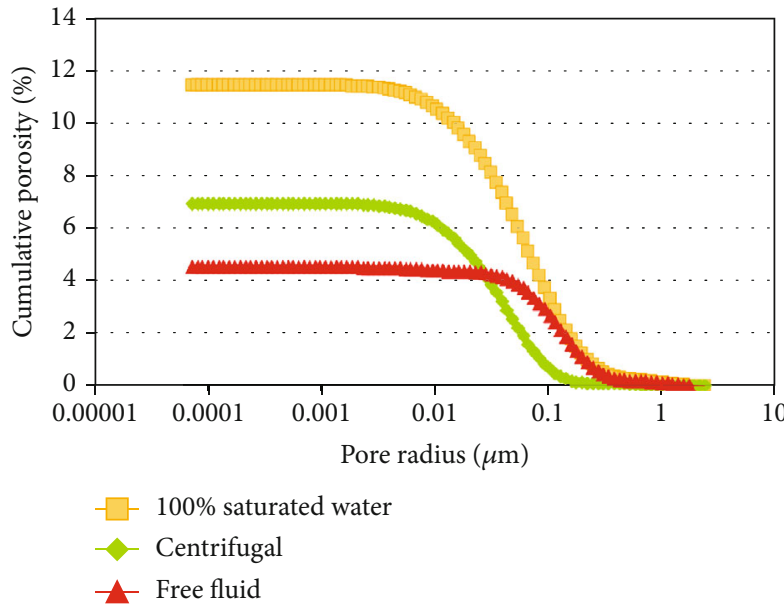

(a)

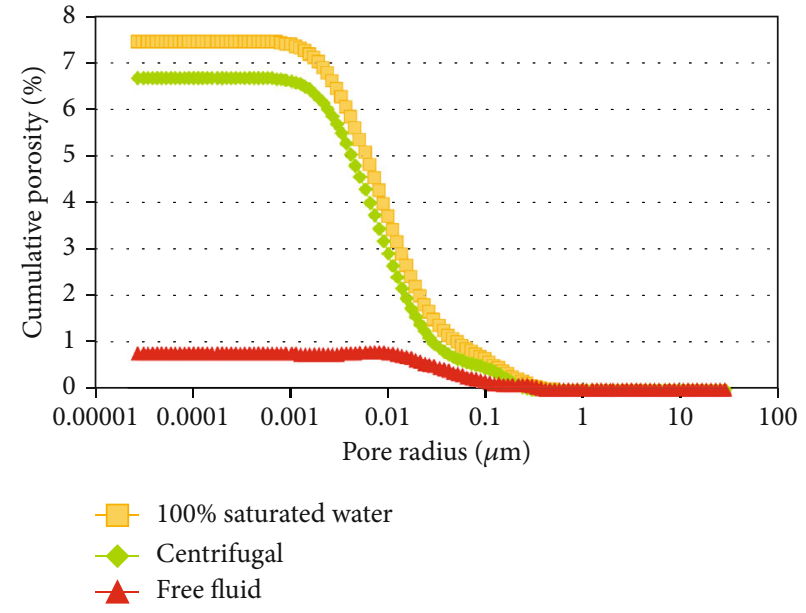

(b)

Figure 7: Calculation results of the free fluid $T_{2}$ spectrum for some typical rock samples: (a) B64-33 and (b) M5-6.

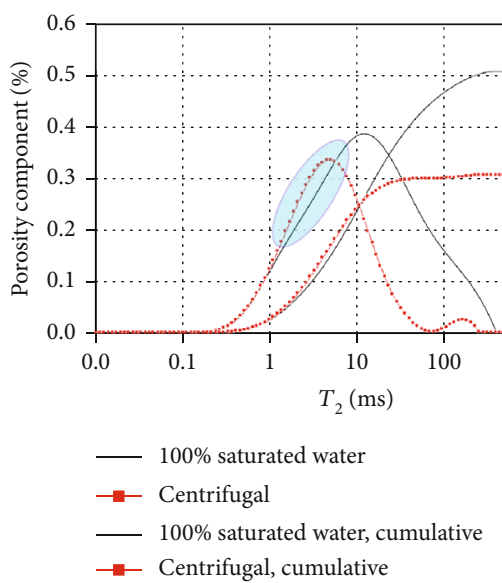

(a)

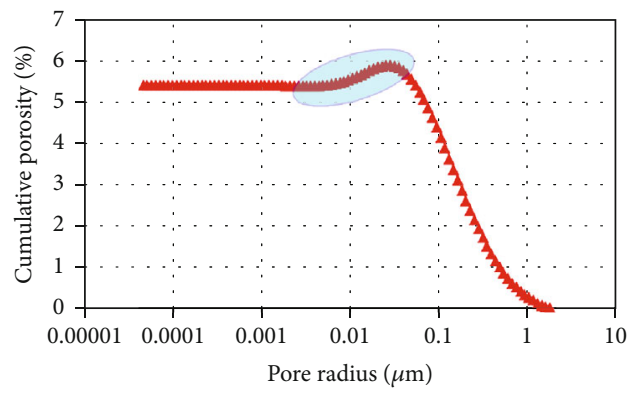

(c)
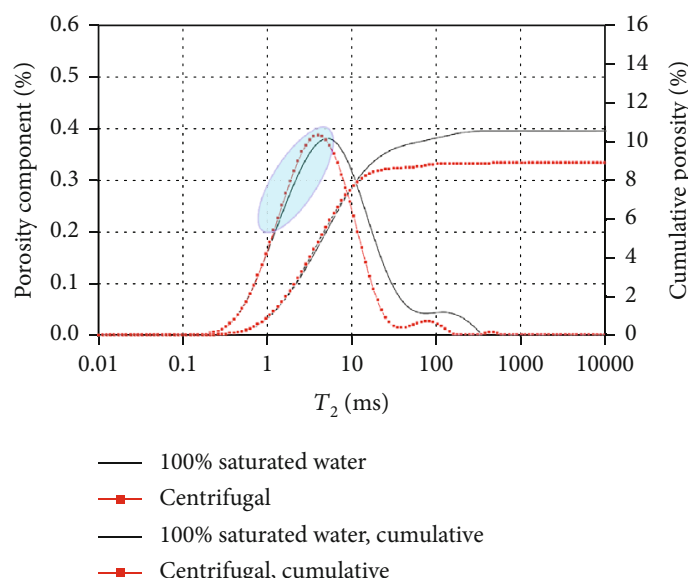

(b)

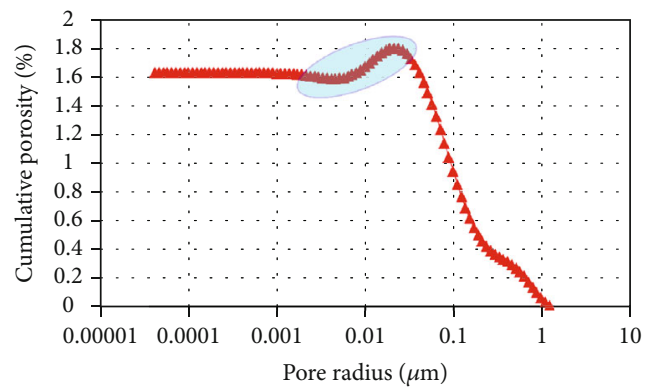

(d)

FIGURE 8: Images showing NMR experimental system errors: (a) B64-38, $T_{2}$ spectrum; (b) B65-8, $T_{2}$ spectrum; (c) B64-38, negative accumulation area; (d) B65-8, negative accumulation area.

3.2. Permeability Prediction. The steps for computation of permeability with the IFU model can be summarized as follows:

(1) Select the fractal units with different parameters for building the IFU model (Table 1), and change the iteration parameters to assure a consistency between the pore diameter distribution spectra of the structural model and the experimental data. Figure 9 reveals fitting results for some representative samples

(2) Based on the completion of the above fitting process, formula (12) can be applied in the IFU model to calculate permeability (Table 2 ) 
TABLe 1: Parameters of the IFU models of some typical samples.

\begin{tabular}{|c|c|c|c|c|c|c|c|c|c|}
\hline Samp & & $\begin{array}{l}\text { Number } \\
\text { of fractal }\end{array}$ & $\begin{array}{l}\text { Number } \\
\text { of pores } \\
\text { generated }\end{array}$ & $\begin{array}{l}\text { Iteration } \\
\text { times }(i)\end{array}$ & $\begin{array}{c}\text { Ratio factor } \\
\text { (b) }\end{array}$ & $\begin{array}{c}\text { Maximum } \\
\text { pore diameter }\end{array}$ & $\begin{array}{l}\text { Number of } \\
\text { units excluded } \\
\text { from iteration }\end{array}$ & $\begin{array}{c}\text { Tortuosity } \\
(\tau)\end{array}$ & $\begin{array}{c}\text { Fractal } \\
\text { dimension }\end{array}$ \\
\hline & Unit A & $1 \times 10^{4}$ & 1 & 3 & 2 & 3.4 & 0 & 1.3336 & 2.5850 \\
\hline & Unit B & $5 \times 10^{5}$ & 2 & 2 & 3 & 0.85 & 0 & 1.6128 & 2.7712 \\
\hline ל & Unit C & $4.5 \times 10^{6}$ & 6 & 2 & 3 & 0.295 & 0 & 1.0684 & 2 \\
\hline & Unit D & $9 \times 10^{7}$ & 2 & 2 & 2 & 0.12 & 0 & 1.1693 & 2 \\
\hline & Unit A & $1 \times 10^{4}$ & 1 & 2 & 2 & 3.3 & 0 & 1.5321 & 2.5850 \\
\hline & Unit B & $3 \times 10^{5}$ & 2 & 2 & 3 & 1.7 & 0 & 1.6128 & 2.7712 \\
\hline DOF' & Unit C & $4 \times 10^{6}$ & 5 & 2 & 3 & 0.57 & 0 & 1.1287 & 2.2619 \\
\hline & Unit D & $1.9 \times 10^{8}$ & 1 & 2 & 3 & 0.22 & 0 & 2.2626 & 2.8928 \\
\hline & Unit A & $1 \times 10^{2}$ & 1 & 2 & 2 & 2.1 & 0 & 1.5321 & 2.5850 \\
\hline & Unit B & $3.5 \times 10^{5}$ & 2 & 3 & 3 & 1.1 & 0 & 1.3932 & 2.7712 \\
\hline $101101-2-2$ & Unit C & $1.8 \times 10^{7}$ & 5 & 3 & 3 & 0.13 & 0 & 1.0532 & 2.2619 \\
\hline & Unit D & $1.2 \times 10^{9}$ & 4 & 2 & 3 & 0.015 & 0 & 1.2190 & 2.4650 \\
\hline & Unit A & $2 \times 10^{2}$ & 1 & 2 & 2 & 1 & 0 & 1.5321 & 2.5850 \\
\hline $95-6$ & Unit B & $1 \times 10^{6}$ & 2 & 3 & 3 & 0.5 & 0 & 1.3932 & 2.7712 \\
\hline & Unit C & $2.7 \times 10^{8}$ & 2 & 2 & 3 & 0.056 & 0 & 1.6128 & 2.7712 \\
\hline & Unit D & 0 & 0 & 0 & 0 & 0 & 0 & - & - \\
\hline
\end{tabular}

In order to make a better comparison between the computed results of the IFU model and the classical models, we subdivide the two classical models into the following four types according to whether the undetermined parameters of the models are constant or not. The relevant parameters of the experiment and models are shown in Tables 3 and 4.

The parameters in Table 3 are substituted into each model in Table 4, and the best model coefficient is determined by programming using the method of three-coefficient regression analysis. It can be seen that in Coates model 1 and SDR model 1 , except that the coefficient is a variable, the index is also not a constant. Actually, when the regression statistical method is used to determine the coefficients and exponents in the equation, due to the lack of data points, the variation range of model parameters is closely related to the selection of initial values, which requires some prior knowledge. Therefore, a more feasible method is to take logarithms on both sides of the equation and then calculate each undetermined coefficient by regression statistical analysis.

Table 5 and Figure 10 show that the classical models cannot accurately calculate the permeability of the sand conglomerate reservoirs in the study area. The average relative error of the results calculated using the IFU model is $23.1 \%$, within a certain tolerance for error, indicating that the IFU model is reliable in simulating the movable fluid pore space based on NMR data.

Also, it is noted that the permeability calculation results, based on the IFU model, are mostly smaller than the experimental ones. This is mainly due to the fact that, for reservoirs with complex pore structures such as sand conglomerate reservoirs, it is difficult to replicate the real conditions of a bound water state in centrifugal experiments. Centrifugal parameter settings often have strong regional characteristics. However, in fact, in order to truly replicate a bound water state and obtain the free fluid $T_{2}$ spectrum, the centrifugal force and centrifugation time must be markedly different for each sample.

Therefore, it is not realistic to increase the centrifugal force and the centrifugation time without reference to other parameters, because "grain shattering" occurs easily during the centrifugation procedure for sand conglomerate rocks. Excessive centrifugal force will damage the samples and then affect the experimental results. Therefore, since it is difficult to accurately measure the content of the movable fluid in centrifugal experiments, the computational accuracy of the IFU model based on NMR data is inevitably somewhat compromised.

\subsection{Other Applications of the IFU Model in Reservoir Physics}

3.3.1. Intermingled Fractal Expression of the Kozeny-Carman Equation. The Kozeny-Carman equation is a semiempirical formula widely used in calculating permeability, whereas the determination of the $\mathrm{KC}$ constant is highly empirical. In fact, the $\mathrm{KC}$ constant is closely related to the microscopic pore structure and is not a fixed value. Since the IFU model can accurately quantify specific pore throat information in the porous medium, the $\mathrm{KC}$ constant, with a practical physical manifestation, can be obtained using the IFU. 
In order to facilitate the presentation of the formula derivation process, this section takes the example of an IFU model with only one fractal unit. Taking tortuosity into consideration, the total internal surface area $\left(A_{\text {in }}\right)$ of the pores produced after the $k$-th iteration is straightforwardly obtained according to equation (3):

$$
A_{\text {in }}=\sum_{i=1}^{k}\left[\left(b^{2}-N_{\text {soild }}-N_{\mathrm{p}}\right)^{i-1} \times N_{\mathrm{p}} \times \pi \times \frac{d_{\max }}{b^{i-1}} \times L_{0} \times \tau\right] \text {. }
$$

Thus, the specific surface $(S)$ of the IFU model is

$$
S=\frac{\pi \tau N_{\mathrm{p}} d_{\max }}{A} \sum_{i=1}^{k} \frac{\left(b^{2}-N_{\text {soild }}-N_{\mathrm{p}}\right)^{i-1}}{b^{i-1}} .
$$

From equation (4), the porosity $(\phi)$ of the IFU model can be rendered as

$$
\phi=\frac{\pi \tau N_{\mathrm{p}} \cdot d_{\max }^{2}}{4 A} \sum_{i=1}^{k} \frac{\left(b^{2}-N_{\text {soild }}-N_{\mathrm{p}}\right)^{i-1}}{b^{2(i-1)}}
$$

where $A$ is the cross-sectional area of the model. Note that for some tight reservoirs with extralow porosity and permeabil- ity, the pore sizes converted using the NMR free fluid $T_{2}$ spectrum are the effective flow radius, without considering boundary layer effects. However, for pore sizes converted by mercury injection capillary pressure (MICP) measurements, it is necessary to deduct the thickness of the boundary layer to obtain an effective flow radius, since mercury does not produce a boundary layer in MICP experiments. For quantitative calculation of the thickness of the boundary layer, please refer to the references made by Tian et al. [40] and Meng et al. [41]. Thus, permeability of the IFU model, taking the thickness of the boundary layer $\left(h_{i}\right)$ into consideration, is given by

$K=\frac{\pi N_{\mathrm{p}}}{128 \tau A} \sum_{i=1}^{k}\left(\left(\frac{d_{\max }}{b^{i-1}-2 h_{i}}\right)^{4} \cdot\left(b^{2}-N_{\text {soild }}-N_{\mathrm{p}}\right)^{i-1}\right)$.

According to the classical Kozeny-Carman equation,

$$
K=\frac{\phi^{3}}{C S^{2}}
$$

where $C$ is the $\mathrm{KC}$ constant. By substituting formulas (15)-(17) in (18), the expression of the $\mathrm{KC}$ constant is obtained:

$$
C=\frac{2 \tau^{2} d_{\max }^{4}\left[\sum_{i=1}^{k}\left(\left(b^{2}-N_{\text {soild }}-N_{\mathrm{p}}\right) / b^{2}\right)^{i-1}\right]^{3}}{\sum_{i=1}^{k}\left(\left(d_{\max } /\left(b^{i-1}-2 h_{i}\right)\right)^{4} \cdot\left(b^{2}-N_{\text {soild }}-N_{\mathrm{p}}\right)^{i-1}\right) \cdot\left[\sum_{i=1}^{k}\left(\left(b^{2}-N_{\text {soild }}-N_{\mathrm{p}}\right) / b\right)^{i-1}\right]^{2}} .
$$

It can be seen that the $\mathrm{KC}$ constant is not an empirical constant without precise physical meaning but is controlled by factors such as iteration parameters, tortuosity, and the thickness of the boundary layer in the IFU model. Once these parameters are given, the value of KC for each sample can be calculated simply by programming.

3.3.2. Intermingled Fractal Expression of Relative Permeability. Based on the IFU model, the expression of relative permeability can also be derived. Similarly to Section 3.3.1, the IFU model with only one fractal unit is taken as an example in this case. The assumptions for the model are as follows:

(a) The reservoir is composed of unequal diameter capillaries with fractal features

(b) The fluid flow conforms to Darcy's law, and the wet phase is distributed in the capillaries whose pore diameters are smaller than the critical capillary radius $\left(r_{c}\right)$ and the nonwet phase is distributed in the capillaries whose pore diameters are larger than the critical capillary radius. Therefore, only one fluid exists in a single capillary

(c) The boundary layer fluid adheres to the inside of the tube wall in the form of a bound water film

(d) The fluid is incompressible, the viscosity is constant, and the percolation process is isothermal

Thus, considering the thickness of the boundary layer, formula (12) can be rewritten as

$$
K=\frac{\pi N_{p}}{128 \tau A} \sum_{i=1}^{k}\left(\left(\frac{d_{\max }}{b^{i-1}-2 h_{i}}\right)^{4} \cdot\left(b^{2}-N_{\text {soild }}-N_{\mathrm{p}}\right)^{i-1}\right) .
$$

According to the definition of saturation,

$$
s_{i}=\frac{V_{i}}{\phi A L_{0}}=\frac{(\pi / 4)\left(\left(d_{\max } / b^{i-1}\right)-2 h_{i}\right)^{2} \cdot L_{0} \cdot \tau \cdot\left(b^{2}-N_{\text {soild }}-N_{\mathrm{p}}\right)^{i-1} \cdot N_{\mathrm{p}}}{\phi A L_{0}} .
$$




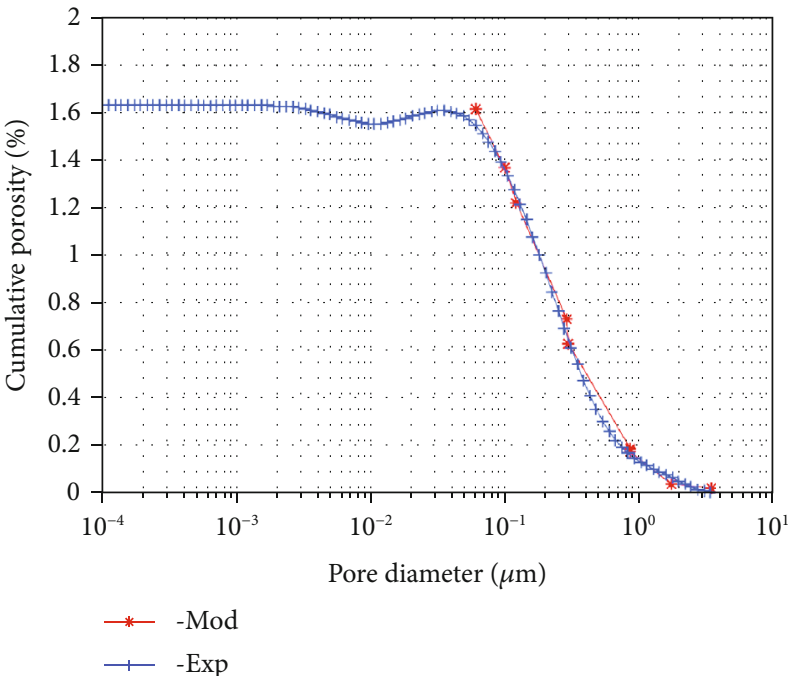

(a)

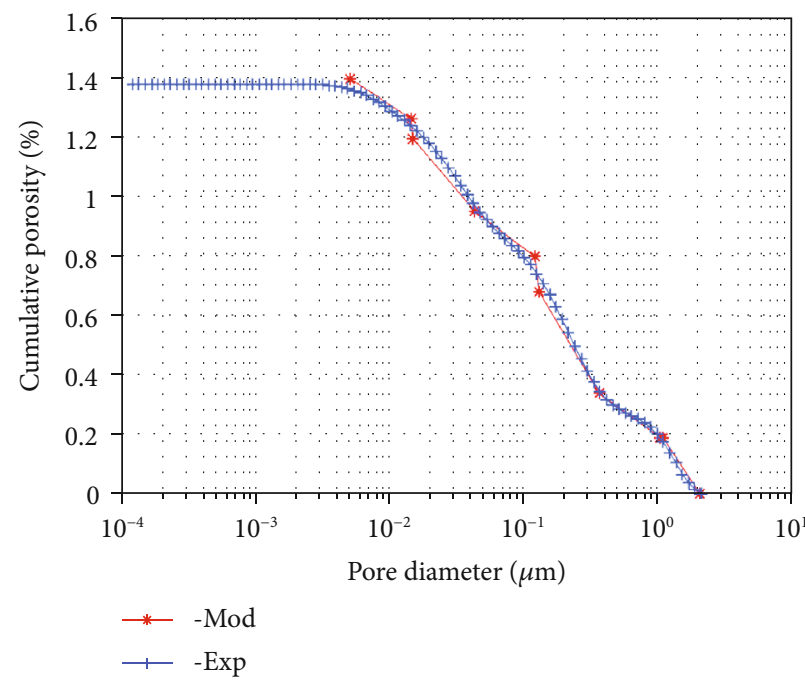

(c)

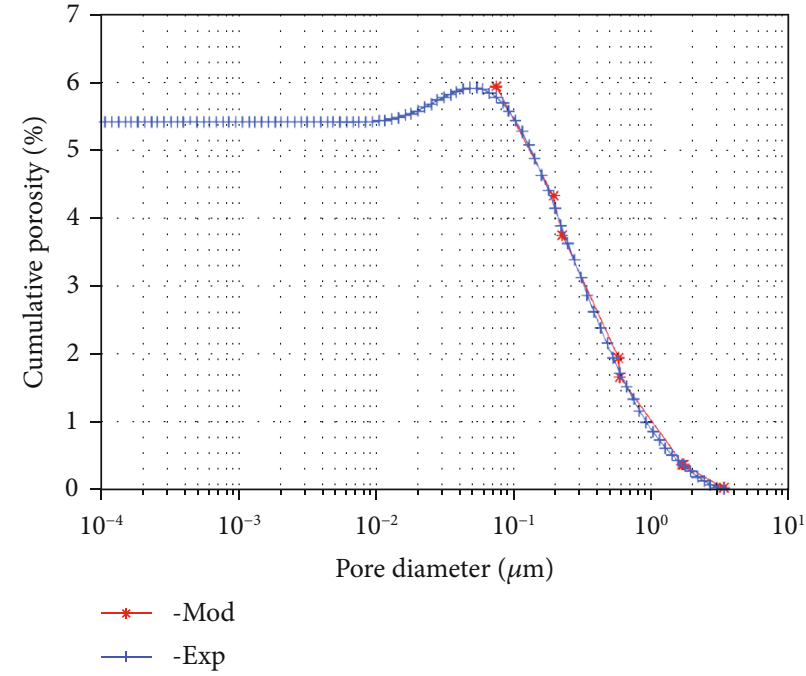

(b)

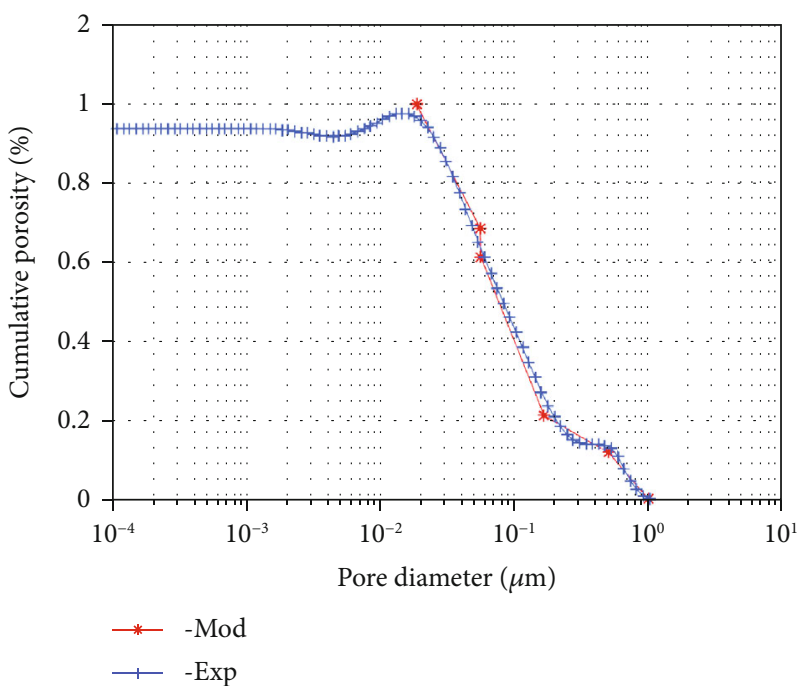

(d)

FIGURE 9: Images showing the IFU model fitting pore size distribution curve of free fluid: (a) B64-3, (b) B64-38, (c) M101-2-2, and (d) M5-6.

TABLE 2: Results of IFU model-computed permeability.

\begin{tabular}{lcc}
\hline Sample ID & $\begin{array}{c}\text { Experimental } \\
\text { permeability }\left(10^{-15} \mathrm{~m}^{2}\right)\end{array}$ & $\begin{array}{c}\text { IFU model-computed } \\
\text { permeability }\left(10^{-15} \mathrm{~m}^{2}\right)\end{array}$ \\
\hline B64-3 & 0.183 & 0.1189 \\
B64-38 & 0.551 & 0.3136 \\
M101-2-2 & 0.034 & 0.0403 \\
M5-6 & 0.0054 & 0.0049 \\
B64-33 & 3.056 & 2.5713 \\
B64-42 & 0.368 & 0.2651 \\
B64-29 & 0.033 & 0.0278 \\
B65-8 & 0.327 & 0.2664 \\
M5-1 & 0.403 & 0.3052 \\
\hline
\end{tabular}

TABLE 3: Parameters of the NMR experiment of some typical samples.

\begin{tabular}{lcccc}
\hline Sample ID & NMR porosity (\%) & FFI $(\%)$ & BVI $(\%)$ & $T_{2 \mathrm{~g}}(\mathrm{~ms})$ \\
\hline B64-3 & 11.2 & 15.31 & 84.69 & 5.562 \\
B64-38 & 13.5 & 39.92 & 60.08 & 11.756 \\
M101-2-2 & 9.4 & 12.86 & 87.14 & 3.362 \\
M5-6 & 7.5 & 10.42 & 89.58 & 3.924 \\
B64-33 & 11.4 & 39.45 & 60.55 & 7.350 \\
B64-42 & 8.4 & 24.16 & 75.84 & 5.284 \\
B64-29 & 12.1 & 17.39 & 82.61 & 5.856 \\
B65-8 & 10.6 & 16.10 & 83.90 & 5.525 \\
M5-1 & 7.8 & 15.76 & 84.24 & 7.031 \\
\hline
\end{tabular}


TABLE 4: Results of undetermined parameters of four models.

\begin{tabular}{lccc}
\hline Model & Expression & Parameter values & Final fitting formula \\
\hline Coates model 1 & $K=n_{1} \phi^{n_{2}}(\mathrm{FFI} / \mathrm{BVI})^{n_{3}}$ & $n_{1}=136.4777, n_{2}=-1.2893, n_{3}=2.6673$ & $K=136.4777 \phi^{-1.2893}(\mathrm{FFI} / \mathrm{BVI})^{2.6673}$ \\
Coates model 2 & $K=C_{1} \phi^{4}(\mathrm{FFI} / \mathrm{BVI})^{2}$ & $C_{1}=1.138 \times 10^{-4}$ & $K=0.000138 \phi^{4}(\mathrm{FFI} / \mathrm{BVI})^{2}$ \\
SDR model 1 & $K=m_{1}(\phi / 100)^{m_{2}} T_{2 \mathrm{~g}}{ }^{m_{3}}$ & $m_{1}=0.0001, m_{2}=-0.2404, m_{3}=3.6672$ & $K=0.0001(\phi / 100)^{-0.2404} T_{2 \mathrm{~g}}{ }^{3.6672}$ \\
SDR model 2 & $K=C_{2}(\phi / 100)^{4} T_{2 \mathrm{~g}}{ }^{2}$ & $C_{2}=24.8333$ & $K=24.8333(\phi / 100)^{4} T_{2 \mathrm{~g}}{ }^{2}$ \\
\hline
\end{tabular}

TABLE 5: Comparison of permeability computed using the IFU and classic models.

\begin{tabular}{lcccccccccc}
\hline Sample & \multicolumn{1}{c}{ IFU } & \multicolumn{9}{c}{ Model-computed permeability and relative error } \\
ID & $\begin{array}{c}\text { Permeability } \\
\left(10^{-15} \mathrm{~m}^{2}\right)\end{array}$ & $\begin{array}{c}\text { Relative } \\
\text { error }(\%)\end{array}$ & $\begin{array}{c}\text { Permeability } \\
\left(10^{-15} \mathrm{~m}^{2}\right)\end{array}$ & $\begin{array}{c}\text { Relative } \\
\text { error }(\%)\end{array}$ & $\begin{array}{c}\text { Permeability } \\
\left(10^{-15} \mathrm{~m}^{2}\right)\end{array}$ & $\begin{array}{c}\text { Relative } \\
\text { error }(\%)\end{array}$ & $\begin{array}{c}\text { Permeability } \\
\left(10^{-15} \mathrm{~m}^{2}\right)\end{array}$ & $\begin{array}{c}\text { Relative } \\
\text { error }(\%)\end{array}$ & $\begin{array}{c}\text { Permeability } \\
\left(10^{-15} \mathrm{~m}^{2}\right)\end{array}$ & $\begin{array}{c}\text { Relative } \\
\mathrm{error}^{(\%)}\end{array}$ \\
\hline B64-3 & 0.1189 & 35.03 & 0.0633 & 65.41 & 0.0585 & 68.03 & 0.1363 & 25.52 & 0.1209 & 33.93 \\
B64-38 & 0.3136 & 43.09 & 1.6004 & 190.45 & 1.6687 & 202.85 & 2.0276 & 267.99 & 1.1400 & 106.90 \\
M101-2-2 & 0.0403 & 18.53 & 0.0461 & 35.59 & 0.0194 & 42.94 & 0.0224 & 34.12 & 0.0219 & 35.59 \\
M5-6 & 0.0049 & 9.26 & 0.0327 & 505.56 & 0.0049 & 9.26 & 0.0418 & 674.07 & 0.0121 & 124.07 \\
B64-33 & 2.5713 & 15.86 & 1.8887 & 38.20 & 0.8159 & 73.30 & 0.3773 & 87.65 & 0.2266 & 92.59 \\
B64-42 & 0.2651 & 27.96 & 0.4153 & 12.85 & 0.0575 & 84.38 & 0.1210 & 67.12 & 0.0345 & 90.63 \\
B64-29 & 0.0278 & 15.76 & 0.0859 & 160.30 & 0.1081 & 227.58 & 0.1616 & 389.70 & 0.1825 & 453.03 \\
B65-8 & 0.2664 & 18.53 & 0.0796 & 75.66 & 0.0529 & 83.82 & 0.1348 & 58.78 & 0.0957 & 70.73 \\
M5-1 & 0.3052 & 24.27 & 0.1105 & 72.58 & 0.0147 & 96.35 & 0.3512 & 12.85 & 0.0454 & 88.73 \\
\hline
\end{tabular}

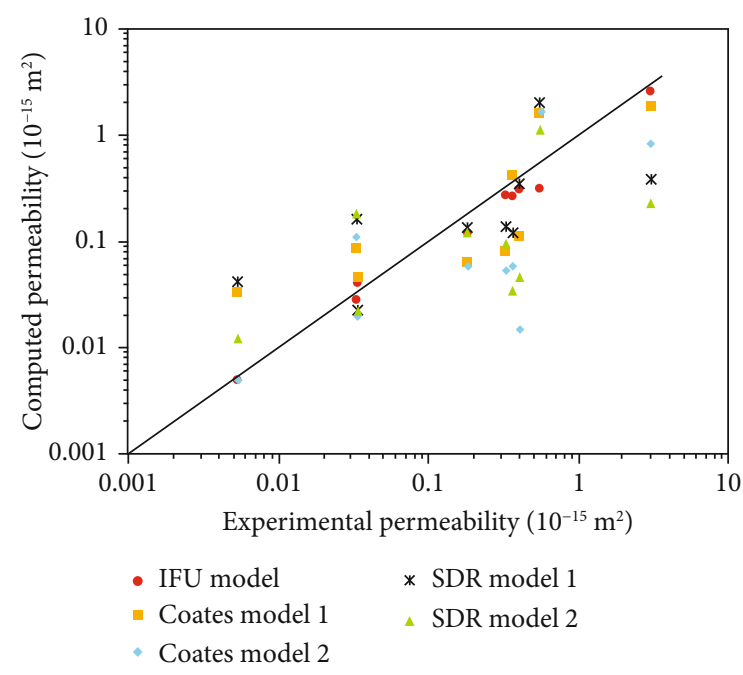

FIgURE 10: Comparison of calculation results from the IFU and the classic models.

The effective permeability can then be rewritten as

$$
K=\frac{\phi}{32 \tau^{2}} \sum_{i=1}^{k} S_{i} \cdot\left(\frac{d_{\max }}{b^{i-1}}-2 h\right)_{i}^{2}
$$

It is indicated from the previous study that, as the iteration time ( $i$ ) increases, the diameters of the generated pores reduce. Therefore, if $c$ is the critical iteration time, then the nonwet phase exists in the pores of $i<c$ and the wet phase exists in the pores of $i \geq c$. by

The effective permeability of the wet phase is then given

$$
K_{\mathrm{w}}=\frac{\phi}{32 \tau_{\mathrm{w}}^{2}} \sum_{i=c}^{k} S_{i} \cdot\left(\frac{d_{\mathrm{max}}}{b^{i-1}}-2 h_{i}\right)^{2}
$$

The effective permeability of the nonwet phase is given by

$$
K_{\mathrm{nw}}=\frac{\phi}{32 \tau_{\mathrm{nw}}{ }^{2}} \sum_{i=1}^{c-1} S_{i} \cdot\left(\frac{d_{\mathrm{max}}}{b^{i-1}}-2 h_{i}\right)^{2} .
$$

The relative permeability of the wet phase can then be derived as follows:

$$
K_{\mathrm{rw}}=\frac{K_{\mathrm{w}}}{K}=\left(\frac{\tau}{\tau_{\mathrm{w}}}\right)^{2} \cdot \frac{\sum_{i=c}^{k} S_{i} \cdot\left(\left(d_{\max } / b^{i-1}\right)-2 h_{i}\right)^{2}}{\sum_{i=1}^{k} S_{i} \cdot\left(\left(d_{\max } / b^{i-1}\right)-2 h_{i}\right)^{2}} .
$$

And the relative permeability of the nonwet phase is

$$
K_{\mathrm{rnw}}=\frac{K_{\mathrm{nw}}}{K}=\left(\frac{\tau}{\tau_{\mathrm{nw}}}\right)^{2} \cdot \frac{\sum_{i=1}^{c-1} S_{i} \cdot\left(\left(d_{\max } / b^{i-1}\right)-2 h_{i}\right)^{2}}{\sum_{i=1}^{k} S_{i} \cdot\left(\left(d_{\max } / b^{i-1}\right)-2 h_{i}\right)^{2}}
$$

where $\tau, \tau_{\mathrm{w}}$, and $\tau_{\mathrm{nw}}$ are the tortuosities of $100 \%$ saturated single-phase, wet-phase, and non-wet-phase fluids, respectively, and they are determined by formula (9) according to their respective saturations. Also, it is found that the forms 
of the formulas are similar to those expressed in the classical Burdine model [42].

Although these derived formulas seem complicated in form, in fact, the IFU model can give detailed quantitative information about each pore throat in the porous medium by fitting the pore size distribution spectrum, which makes the calculations of these parameters (permeability, KC constant, relative permeability, etc.) rapid and convenient by computer programming.

\section{Conclusions}

(1) The classical Coates and SDR models cannot fulfill the precision requirements of permeability calculations for sand conglomerate reservoirs in the study area, and the calculation accuracy of results based on the IFU model is significantly improved. Also, the model-computed results are slightly smaller than the experimental ones, because it is difficult to accurately measure the content of the movable fluid in the centrifugal experiment

(2) The new intermingled fractal expressions of the Kozeny-Carman equation and relative permeability were derived based on the IFU model, and the physical meanings of the parameters were explained. The expressions appear complicated in form but are easy to calculate and apply using computer programming, since their iteration parameters are defined

(3) The authors believe that the application of the IFU model in reservoir physics is much more than that, and further exploration of possible improvements and applications of the model is necessary and meaningful

\section{Data Availability}

All data generated or analyzed during this study are included in this article, and all data included in this study are available upon request by contact with the corresponding author.

\section{Conflicts of Interest}

The authors declare that there is no conflict of interest regarding the publication of this paper.

\section{Acknowledgments}

This research was financially supported by the National Key Basic Research and Development Program (973 Program), China (2014CB239000), the China National Science and Technology Major Projects (2017ZX05001), and the PetroChina Science and Technology Major Projects (2016B-0304).

\section{References}

[1] C. Z. He and M. Q. Hua, "Fractal geometry description of reservoir pore structure," Oil and Gas Geology, vol. 19, no. 1, pp. 15-23, 1998.
[2] L. R. Li, S. Y. Yuan, and Y. L. Hu, "A new model for describing the relationship between the permeability and the porosity of fractal porous media," Journal of Xi'an Shiyou University (Natural Science Edition), vol. 25, no. 3, pp. 49-51, 2010.

[3] B. Zheng and J. H. Li, "A new fractal permeability model for porous media based on Kozeny-Carman equation," Natural Gas Geoscience, vol. 26, no. 1, pp. 193-198, 2015.

[4] R. T. Bai, Z. P. Li, J. X. Nan, F. P. Lai, H. Li, and Q. Wei, “The fractal permeability model in tight sand reservoir accounts for start-up gradient," Natural Gas Geoscience, vol. 27, no. 1, pp. 142-148, 2016.

[5] K. Li, "Analytical derivation of brooks-Corey type capillary pressure models using fractal geometry and evaluation of rock heterogeneity," Journal of Petroleum Science and Engineering, vol. 73, no. 1-2, pp. 20-26, 2010.

[6] H. Y. Liu, Z. Y. Tian, and Z. Y. Xu, "Quantitative evaluation of carbonate reservoir pore structure based on fractal characteristics," Lithologic Reservoirs, vol. 29, no. 5, pp. 97-105, 2017.

[7] F. Wang, L. Jiao, Z. Liu, X. Tan, C. Wang, and J. Gao, "Fractal analysis of pore structures in low permeability sandstones using mercury intrusion porosimetry," Journal of Porous Media, vol. 21, no. 11, pp. 1097-1119, 2018.

[8] F. Wang, K. Yang, and J. Cai, "Fractal characterization of tight oil reservoir pore structure using nuclear magnetic resonance and mercury intrusion porosimetry," Fractals, vol. 26, no. 2, article 1840017, 2018.

[9] Q. H. Zhao, Z. P. Li, F. P. Lai, and Y. Du, "Fractal characteristics of rock pores in shale gas reservoir," Journal of Liaoning Technical University (Natural Science Edition), vol. 35, no. 9, pp. 914-919, 2016.

[10] L. Q. Sima, G. D. Yang, F. Wu, L. Wang, and F. Meng, "Fractal feature about the pore structure and controlling factor in tight glutenite reservoir in Baikouquan formation of Mahu depression in Junggar Basin," Well Logging Technology, vol. 40, no. 5, pp. 609-616, 2016.

[11] D. Y. Li, S. B. Zang, X. J. Ren, H. J. Hui, H. Wang, and F. F. Miao, "Study on pore structure of low permeability reservoirs with fractal theory," Liaoning Chemical Industry, vol. 39, no. 7, pp. 723-726, 2010.

[12] J. Lai, G. W. Wang, Y. Q. Zheng, W. Y. Li, and C. Cai, "Method for calculating the fractal dimension of the pore structure of low permeability reservoirs: a case study on the Xujiahe formation reservoir in Central Sichuan basin," Journal of Northeast Petroleum University, vol. 37, no. 1, pp. 1-7, 2013.

[13] J. Z. Zhang, Fractal (Second Edition), Tsinghua University Press, Beijing, 2011.

[14] Y. Zhou, S. Wu, Z. Li et al., "Multifractal study of threedimensional pore structure of sand-conglomerate reservoir based on CT images," Energy \& Fuels, vol. 32, no. 4, pp. 4797-4807, 2018.

[15] T. F. Zhang, S. Y. Xie, Z. Y. Bao, Y. Wang, and L. Y. Pan, "Fractal and multifractal research on pore system for porous dolomite reservoirs based on high-resolution CT," Geological Science and Technology Information, vol. 35, no. 6, pp. 5562, 2016.

[16] G. Pia, L. Casnedi, M. Ionta, and U. Sanna, "On the elastic deformation properties of porous ceramic materials obtained by pore-forming agent method," Ceramics International, vol. 41, no. 9, pp. 11097-11105, 2015.

[17] G. Pia, L. Casnedi, and U. Sanna, "Porosity and pore size distribution influence on thermal conductivity of yttria-stabilized 
zirconia: experimental findings and model predictions," Ceramics International, vol. 42, no. 5, pp. 5802-5809, 2016.

[18] G. Pia and L. Casnedi, "Heat transfer in high porous alumina: experimental data interpretation by different modelling approaches," Ceramics International, vol. 43, no. 12, pp. 9184-9190, 2017.

[19] G. Pia and U. Sanna, "A geometrical fractal model for the porosity and thermal conductivity of insulating concrete," Construction and Building Materials, vol. 44, pp. 551-556, 2013.

[20] G. Pia and U. Sanna, "An intermingled fractal units model to evaluate pore size distribution influence on thermal conductivity values in porous materials," Applied Thermal Engineering, vol. 65, no. 1-2, pp. 330-336, 2014.

[21] G. Pia and U. Sanna, "An intermingled fractal units model and method to predict permeability in porous rock," International Journal of Engineering Science, vol. 75, pp. 31-39, 2014.

[22] G. Pia, C. Siligardi, L. Casnedi, and U. Sanna, "Pore size distribution and porosity influence on sorptivity of ceramic tiles: from experimental data to fractal modelling," Ceramics International, vol. 42, no. 8, pp. 9583-9590, 2016.

[23] G. Pia, "High porous yttria-stabilized zirconia with aligned pore channels: morphology directionality influence on heat transfer," Ceramics International, vol. 42, no. 10, pp. 1167411681, 2016.

[24] X. H. Tan, J. Y. Liu, X. P. Li, L. H. Zhang, and J. Cai, “A simulation method for permeability of porous media based on multiple fractal model," International Journal of Engineering Science, vol. 95, pp. 76-84, 2015.

[25] Z. L. Chen, N. T. Wang, L. Sun, X. H. Tan, and S. Deng, "Prediction method for permeability of porous media with tortuosity effect based on an intermingled fractal units model," International Journal of Engineering Science, vol. 121, pp. 8390, 2017.

[26] X.-H. Tan, X.-P. Li, J.-Y. Liu, L.-H. Zhang, and J. Cai, "Fractal analysis of stress sensitivity of permeability in porous media," Fractals, vol. 23, no. 2, article 1550001, 2015.

[27] C. Li, M. Lin, L. Ji, W. Jiang, and G. Cao, "Investigation of intermingled fractal model for organic-rich shale," Energy \& Fuels, vol. 31, no. 9, pp. 8896-8909, 2017.

[28] C. Li, M. Lin, L. Ji, W. Jiang, and G. Cao, "Rapid evaluation of the permeability of organic-rich shale using the $3 \mathrm{D}$ intermingled-fractal model," SPE Journal, vol. 23, no. 6, pp. 2175-2187, 2018.

[29] B. B. Mandelbrot, The Fractal Geometry of Nature, Freeman, New York, 1983.

[30] G. R. Coates, J. Galford, and D. Mardon, "A new characterization of bulk-volume irreducible using magnetic resonance," Logic and Analysis, vol. 39, no. 1, pp. 51-63, 1998.

[31] W. E. Kenyon, P. I. Day, C. Straley, and J. F. Willemsen, “A three-part study of NMR longitudinal relaxation properties of water-saturated sandstones," SPE Formation Evaluation, vol. 3, no. 3, pp. 622-636, 2013.

[32] Z. G. Cheng, S. C. Luo, Z. W. Du, S. Chang, G. L. Li, and H. Li, "The method to calculate tight sandstone reservoir permeability using pore throat characteristic parameters," Well Logging Technology, vol. 38, no. 2, pp. 185-189, 2014.

[33] L. Z. Zou and Q. H. Liu, "Analysis of permeability model of nuclear magnetic resonance logging," World Well Logging Technology, vol. 183, no. 3, pp. 27-31, 2011.
[34] M. S. Zhou, J. Yin, J. L. Pan, S. L. Ren, S. X. Yang, and B. Z. Liu, "Method for effectiveness evaluation of low permeability reservoir based on NMR logging pore," World Well Logging Technology, vol. 38, no. 4, pp. 452-457, 2014.

[35] Y. Yao, D. Liu, Y. Che, D. Tang, S. Tang, and W. Huang, "Petrophysical characterization of coals by low-field nuclear magnetic resonance (NMR)," Fuel, vol. 89, no. 7, pp. 1371-1380, 2010.

[36] Y. Bo-Ming and L. Jian-Hua, "A geometry model for tortuosity of flow path in porous media," Chinese Physics Letters, vol. 21, no. 8, pp. 1569-1571, 2004.

[37] M. J. Yun, M. B. Yu, P. Xu, and J. S. Wu, "Geometrical models for tortuosity of streamlines in three-dimensional porous media," Canadian Journal of Chemical Engineering, vol. 84, no. 3, pp. 301-309, 2006.

[38] Y. B. Yao, D. M. Liu, Y. D. Cai, and J. Q. Li, "Fine quantitative characterization of pores and fractures of coal based on NMR and X-CT," Scientia Sinica Terrae, vol. 40, no. 11, pp. 1598$1607,2010$.

[39] C. X. Ning, Z. X. Jiang, Z. Y. Gao et al., "Quantitative evaluation of pore connectivity with nuclear magnetic resonance and high pressure mercury injection: a case study of the lower section of Es3 in Zhanhua sag," Journal of China University of Mining and Technology, vol. 46, no. 3, pp. 578-585, 2017.

[40] X. F. Tian, L. S. Cheng, X. L. Li et al., "A new method to calculate relative permeability considering the effect of pore-throat distribution," Journal of Shaanxi University of Science \& Technology (Natural Science Edition), vol. 6, pp. 100-104, 2014.

[41] Q. Meng, H. Liu, and J. Wang, "A critical review on fundamental mechanisms of spontaneous imbibition and the impact of boundary condition, fluid viscosity and wettability," Advances in Geo-Energy Research, vol. 1, no. 1, pp. 1-17, 2017.

[42] N. T. Burdine, "Relative permeability calculation from size distribution data," Transactions of AIME, vol. 198, pp. 71-78, 1953. 